\title{
Industrial Applications of Fourier Transform In Engineering Field
}

\author{
Nikita Kishor Chaudhari, Amol D. Lokhande, MeghaKishorKothawade \\ School of Engineering \& Technology, Sandip University, Mahiravani, Nashik, Maharashtra, India. \\ *Corresponding author Email: nikitachaudhari0300@gmail.com
}

\begin{abstract}
Fourier Transform is valuable in the investigation of arrangement of fractional differential condition to address introductory initial boundary value problems. A Fourier Transform when applied to partial differential equation decreases the quantity of free factors by one.We utilize Fourier Transform in signal \&image processing. It is additionally valuable in PDAs, LTI framework and circuit investigation.Fourier transform in different applications has expanded in recent years. This transform is one of the easiest changeamong the other change strategy utilized in science. The time utilization is lesser because of this strategy. It has immense use in power dissemination framework, mechanical framework, enterprises and remote organizations. Chiefly in power dispersion framework, it is the moderation of force quality unsettling influence required quick, precise and high commotion invulnerable technique. This paper has huge of its applications in the fields of clinical science and the utilization of it in the cell phone. In mobile phone, the Fourier transform utilizes the sign handling structures and the creation of the cell phone. This paper incorporates the meaning of Fourier transform and it properties through which the arrangement of the issue will be simpler than anticipated. The Fourier transform is very significant for the cutting edge world for the simpler arrangement of the issues. This paper audit the strength of Fourier transform, in ongoing year interest of this strategy and its utilization in various field and their applications.
\end{abstract}

Keywords: Fourier transforms, Fourier sine and cosine Transforms, Fourier transforms, signal processing, cell phone.

\section{Introduction}

Fourier transform is a process that transforms a function from its time domain to its frequency domain. It was first used by Jean Baptist Fourier in 1768. The Fourier transform is a widely used math tool that enables one to decompose a function into its sinusoidal basis functions. It is also used to analyse data and develop computer models. Compelling and productive picture handling calculations for specific areas of issues are troublesome due to the customary portrayal of picture as power at a position, which neglects to catch that pixel's relationship with different pixels and shadings in the picture. Numerous numerical ideas that this paper depends on will be depicted re-exhaustively in the accompanying segments. Just working information on ideas up to analytics is accepted. I'm expecting that many fields and many interests will be addressed in the class, and this raises an significant issue for us all to know about. With the variety of interests and foundations present not all models and applications will be natural and of importance to all individuals. We'll all need to cut each other a little room to breadth, and it's an opportunity for us all to stretch out. Similarly, it's additionally significant for you to understand that this is one seminar on the Fourier change among numerous potential courses. The extravagance of the subject, both numerically and in the scope of uses, implies that we'll simply decide continually. Books regarding the matter don't resemble the other the same, nor do they resemble these notes - even the documentation utilized for essential items and activities can shift from one book to another. I'll attempt to call attention to when a certain decision takes us along a specific way, and I'll attempt to offer something of what the substitute ways maybe. The Fourier Transform is a speculation of the Fourier series. It just applies to ceaseless and an occasional capacities. We characterized Fourier Transform of a piecewise ceaseless and totally integrable capacity $x(t)$ by,

$$
\mathrm{X}(\omega)=\mathrm{F}\{\mathrm{x}(\mathrm{t})\}=\int \infty x(t) \cdot e-\mathrm{j} \omega t \mathrm{dt}
$$

In this paper, we explore the use of the fragmentary Fourier change to 2 distinct areas of picture handling and feature the advantages of these applications.

\section{History}

In arithmetic, a Fourier Transform (FT) is a numerical transform that decays functions relying upon frequency or time into functions relying upon spatial or fleeting frequency, like the outflow of a melodic harmony as far as the volumes and frequencies of its constituent notes. The term Fourier transform alludes to both the frequencydomain and the numerical activity that relates the frequency domain to an element of room or time. The Fourier transform of an element of time is a complex-esteemed function of frequency, whose greatness (outright worth) addresses how much that frequency present in the first function, and whose contention is the stage counterbalanced of the fundamental sinusoid in that frequency. The Fourier transform isn't restricted to elements of time, yet the space of the first function is regularly alluded to as the time area. There is additionally a reverse Fourier transform that numerically integrates the first function from its frequency domain, as demonstrated by the Fourier reversal hypothesis. The Fourier series is named out of appreciation for JeanBaptiste Joseph Fourier (1768-1830), who made significant commitments to the investigation of mathematical series, after 
fundamental examinations by Leonhard Euler, Jean le Rondd'Alembert, and Daniel Bernoulli. Fourier presented the series to address the hotness condition in a metal plate, distributing his underlying outcomes in his 1807 Mémoiresur la spread de la chaleurdans les corps solides (Treatise on the engendering of hotness in strong bodies), and distributing his Théorieanalytique de la chaleur (Analytical hypothesis of heat) in 1822. The Mémoire presented Fourier examination, explicitly Fourier series. Through Fourier's exploration the reality was set up that a subjective (from the beginning, persistent and later summed up to any piecewise-smooth) transform can be addressed by a geometrical series. The main declaration of this incredible disclosure was made by Fourier in 1807, preceding the French Academy. Early thoughts of breaking down an occasional function into the amount of straightforward wavering functions date back to the third century $\mathrm{BC}$, when old cosmologists proposed an empiric model of planetary movements, in light of deferents and epicycles. One inspiration for the Fourier transform comes from the investigation of Fourier series. In the investigation of Fourier series, convoluted yet occasional functions are composed as the amount of straightforward waves numerically addressed by sines and cosines. The Fourier transform is an augmentation of the Fourier series that outcomes when the time of the addressed function is stretched and permitted to move toward limitlessness.

The Fourier change is a numerical change that proselytes between two portrayals of a signal:

1. The time-domain, or the worth of the sign as an element of time.

2. The frequency domain, orthe sufficiency and period of the sign asan element of frequency.

Note that stage has a similar definition here as it accomplishes for waves - it is the point counterbalanced at which we start drawing a wave. We can express the (consistent) Fourier transform F of a function $\mathrm{f}: \mathrm{R} \rightarrow \mathrm{C}$. Functions that are limited in the time space have Fourier transforms that are fanned out across the frequency domain as well as the other way around, a peculiarity known as the vulnerability standard. The basic case for this standard is the Gaussian function, vital in likelihood hypothesis and insights just as in the investigation of actual peculiarities displaying ordinary dissemination (e.g., dispersion). The Fourier transform of a Gaussian function is another Gaussian function. Joseph Fourier presented the transform in his investigation of heat move, where Gaussian functions show up as arrangements of the heat equation.

\section{Preliminaries}

The Discrete Fourier Transform is firmly connected with Discrete Fourier series and the Fourier Transform. The Discrete Fourier change is the fitting Fourier portrayal for advanced PC acknowledgment since it is discrete and of limited length in both time and recurrence area.Likewise the Fast Fourier Transform registers DFT and delivers the very same outcome as assessing DFT definition straightforwardly .It is a lot quicker than DFT. Assuming that we neglect to reuse what we as of now have, we end up in a dilemma of running out of assets. By decreasing our waste, we are rationing our assets. Assets, for example, aluminum, oil and wood are totally used to make new items, for example, jars, plastic sacks and paper buildling.Project Review Traditional estimation frameworks might have two hubs sword for functional directors. To an ever increasing extent organizations are utilizing a steady scorecard of execution to recognize and impart key factors that advance better pointers request and future worth. It is able. Inside the above definition are significant speculations of approach. In the first place,the revolutionary and moderate standard expanding. PPR arranged generally speaking, manual explicitly for consider or lock in rebuilding. The primary commitment of the book is to give a far reaching and complete arrangement of directions that are not seen as in quite a large number "rebuilding" books. The inspiration for rebuilding is generally the aftereffect of a change, which ought to be followed up on by a ranking director or sponsorl. Inspiration might be the deficiency of portion of the overall industry, apprehension about forceful contenders or the objective of extending the stock or entering new business sectors. Rebuilding is characterized here as "the extremist and fast overhaul of key, esteem added business processes - and the frameworks, political and hierarchical constructions that support them - further developing work processes and usefulness inside an association." This part contains a contextual investigation that presents and talks about the aftereffects of a business concentrate on connected with designing alumni.

PropertiesofFourierTransform: Properties of the Fourier change work with the change from the time area to recurrence space as well as the other way around.

$>$ Linearity:-The Fourier Transform fulfils linearity and standard of superposition Consider two functions x1(t) \& $\mathrm{x} 2(\mathrm{t})$,

$\operatorname{IfF}[\mathrm{x} 1(\mathrm{t})]=\mathrm{X} 1(\omega), \mathrm{F}[\mathrm{x} 2(\mathrm{t})]=\mathrm{X} 2(\omega)$.

ThenF $[\mathrm{a} 1 \mathrm{x} 1(\mathrm{t})+\mathrm{a} 2 \times 2(\mathrm{t})]=\mathrm{a} 1 \mathrm{X} 1(\omega)+\mathrm{a} 2 \mathrm{X} 2(\omega)$.

$>$ Scaling:-

$\mathrm{F}[(\mathrm{x})]=\mathrm{X}(\omega)$ If $\mathrm{a}$ is real constant thenF[x (at) $]=1 /|\mathrm{a}| \mathrm{X}(\omega) \mathrm{m}$.

$>$ Symmetry:-

If $F[x(t)]=X(\omega)$ then $X(t)=2 \pi X(-\omega)$. 
$>$ Convolution:-Fourier change makes the convolution of two signals into the result of their Fourier Transforms. There are two sorts of convolution properties, one for time domain and other for frequency domain.

$>$ Time Convolution:-

If $\mathrm{F}[\mathrm{x} 1(\mathrm{t})]=\mathrm{X} 1(\omega), \mathrm{F}[\mathrm{x} 2(\mathrm{t})]=\mathrm{X} 2(\omega)$ Then $\mathrm{Y}(\omega)=\mathrm{X} 1(\omega) . \mathrm{X} 2(\omega)$.

$>$ Frequency Convolution:-

$(t) \cdot x(t)=1 / 2 \quad X(\omega) * X(\omega)$

$>$ Shifting Property:-

$\mathrm{x}(\mathrm{t}-\mathrm{t} 0)=e-\mathrm{j} \omega t 0 \mathrm{X}(\omega)$

Duality:-

$\mathrm{X}(\mathrm{t})=2 \pi \mathrm{x}(-\omega)$

$>$ Differentiation:-

$>$ Connection between Fourier Transform \&Laplace Transform:-

We knowthat

$\mathrm{X}(\omega)=\mathrm{F}\{\mathrm{x}(\mathrm{t})\}=\int \infty x(t) \cdot e-\mathrm{j} \omega t \mathrm{~d} t$

By using Laplace Transform,

$\mathrm{X}(s)=\int \infty x(t) \cdot e^{-} s t \mathrm{~d} t$

Here we can say that Fourier Transform is a unique instance of Laplace Transform where ( $s=\mathrm{j} \omega)$

i.e. $X(\mathrm{~s}) \mathrm{s}=\mathrm{j} m=\mathrm{F}\{\mathrm{x}(\mathrm{t})\}$.

\section{Applications Of Fourier Transform}

The Fourier Transform method is used in many fields of Industry \& Engineering field such as

1. Application to IBVP

2. Circuit Analysis

3. Signal Analysis

4. Cell phones

5. Image Processing

6. Signal Processing\& LTI system

Application to Initial Boundary Value Problems(IBVP) : The arrangement of an IBVP comprises of an partial differential equation along with boundary and initial conditions can be tackled by Fourier Transform strategy. Here we tackle the heat condition scientifically by utilizing boundary condition. For this situation fractional differential conditions lessens to an Ordinary Differential Equations in Fourier Transform which is addressed. A boundary condition that indicates the worth of the actual capacity is a Dirichlet boundary condition or first-type boundary condition. For instance, on the off chance that one finish of an iron bar is held at outright zero, the worth of the issue would be known by then in space. A boundary condition that indicates the worth of the typical subordinate of the capacity is a Neumann boundary condition or second-type boundary condition. For instance, on the off chance that there is a warmer toward one side of an iron bar, energy would be added at a consistent rate yet the real temperature would not be known. A two-point boundary value problem (BVP) of total order $\mathrm{n}$ on a finite interval $[\mathrm{a}, \mathrm{b}]$ may be written as an explicit first order system of ordinary differential equations (ODEs) with boundary values evaluated at two points as,

$$
y^{\prime}(x)=f(x, y(x)), x \in(a, b), \quad g(y(a), y(b))=0
$$

Circuit Analysis:This work looks at the examination of electric circuits and the portrayal of occasional capacities as limitless mathematically series in sine and cosine terms (or complex exponentials), and presents the essential investigation of Fourier series concerning its applications in electric circuits. A huge extent of peculiarities contemplated in designing and science, for example, substituting current and voltage are intermittent in nature, and can be examined into their constituent parts (for example essentials and music) by utilization ofFourier series. . The electric occasional signs are disintegrated into

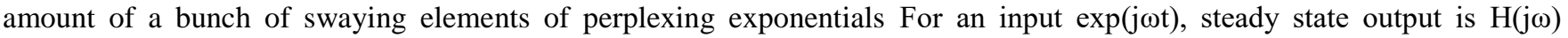

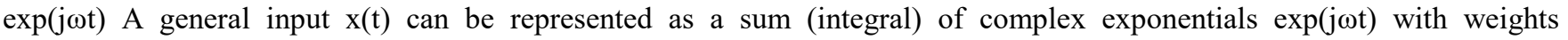
$X(j \omega) \mathrm{d} \omega / 2 \pi$,

$x(t)=\frac{1}{2 \pi} \int_{-\infty} \ldots x(j \omega) \exp (j \omega t)$ 


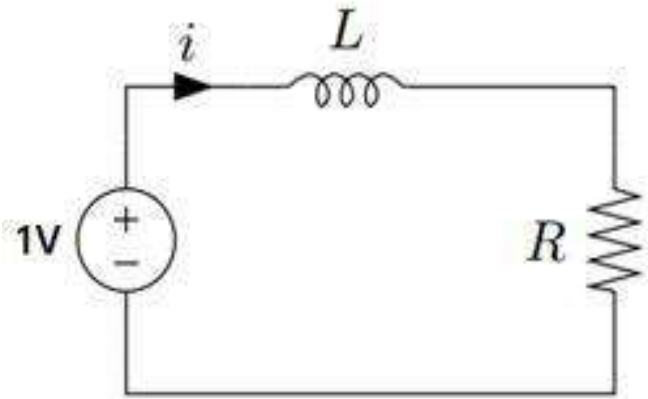

initial current: $i(0)$

Signal Analysis: Signal handling is the investigation, understanding, and control ofany time shifting amount . Signs of interest incorporate sound records, pictures, radar, and organic signals. Possibilities for application in this space are tremendous, and they incorporate pressure, sound decrease, signal grouping, furthermore discovery of dark examples. Maybe the most famous instrument for signal handling is Fourier investigation, which deteriorates a capacity into an amount of sinusoidal premise capacities. For signals whose frequencies change on schedule, Fourier examination has inconveniences which can be overwhelmed by utilizing a windowing interaction called the Short Term Fourier Transform. The windowing interaction can be better further utilizing wavelet examination. This paper will depict every one of these cycles exhaustively, and will apply a wavelet investigation to Pasco climate information. This application will endeavor to boundary temperature variances and how they have changed starting around 1970. The Fourier transform is a numerical formula that relates a signal sample on time or space to a similar signal examined in frequency. In signal handling, the Fourier transform can uncover significant characteristics of a signal, to be specific, its frequency components.

$$
y_{k+1}=\sum_{j=0}^{n-1} \omega^{j k} x_{j+1} . \quad f(x)=a_{0}+\sum_{k=1}^{\infty} a_{k} \sin (k x)+\sum_{k=1}^{\infty} b_{k} \cos (k x) .
$$

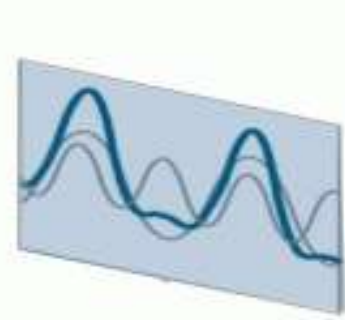

Time Domain $s(t)$
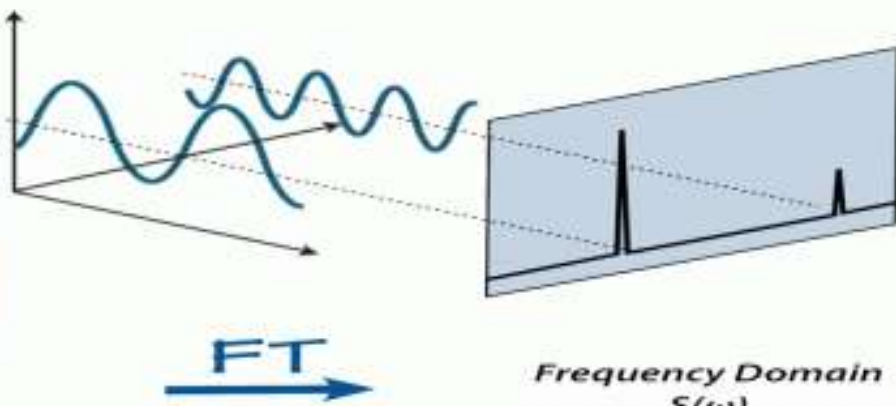

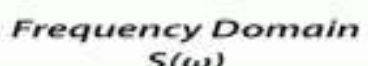
S(w)

Cell phones: Communication is completely founded on Mathematics .The communication incorporates programmed transmission of information over wires furthermore radio circuits through signals.Cell phones are one of the most noticeable specialized gadget, the mobile phone is significantly changing the manner in which individuals cooperate and speak with one another. The standard of Fourier Transform is utilized in signal, ,for example, sound delivered by an instrument For e.gpiano, violin ,drum any sound recording can be addressed as the amount of an assortment of sine and cosine waves with different frequencies and amplitudes. This assortment of waves can then be controlled without hardly lifting a finger. Our cell phone has performing Fourier Transform. Each cell phone -, for example, netbook, tablet , and telephone have been underlying high speed cell association, very much like Fourier Transform. People effectively perform it precisely ordinary. For ex. At the point when you are in a room with a lot of clamor and you specifically hear your name over the commotion, then, at that point, you just performed Fourier change.

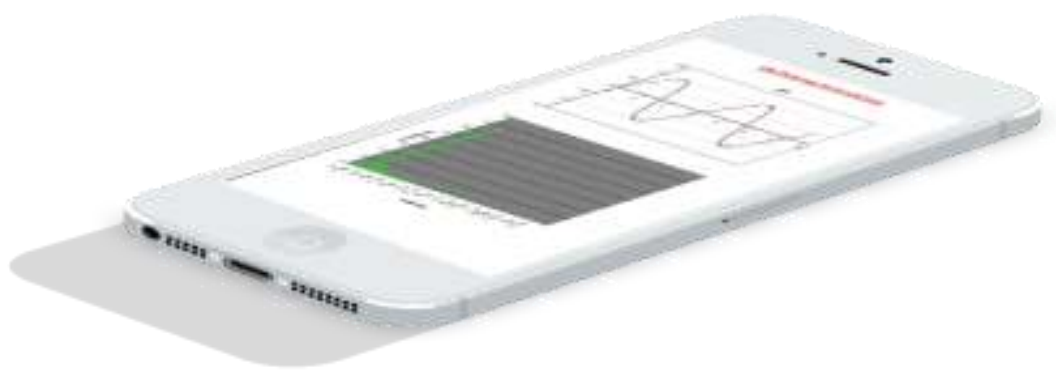


Image/Picture Processing: Fourier transform is utilized in a wide scope of uses, for example, picture examination, picture separating, picture recreation what's more picture pressure. The Fourier Transform is a significant picture handling instrument which is utilized to break down a picture into its sine and cosine parts. The Fourier Transform is utilized to get to the mathematical qualities of a spatial space picture. Since the picture in the Fourier area is deteriorated into its sinusoidal parts, it is not difficult to inspect or process certain frequencies of the picture, subsequently impacting the mathematical design in the spatial space.In many executions the Fourier picture is moved so that the DC-value (for example the picture mean) $F(0,0)$ is shown in the focal point of the picture. The further away from the middle a picture point is, the higher is its relating frequency.

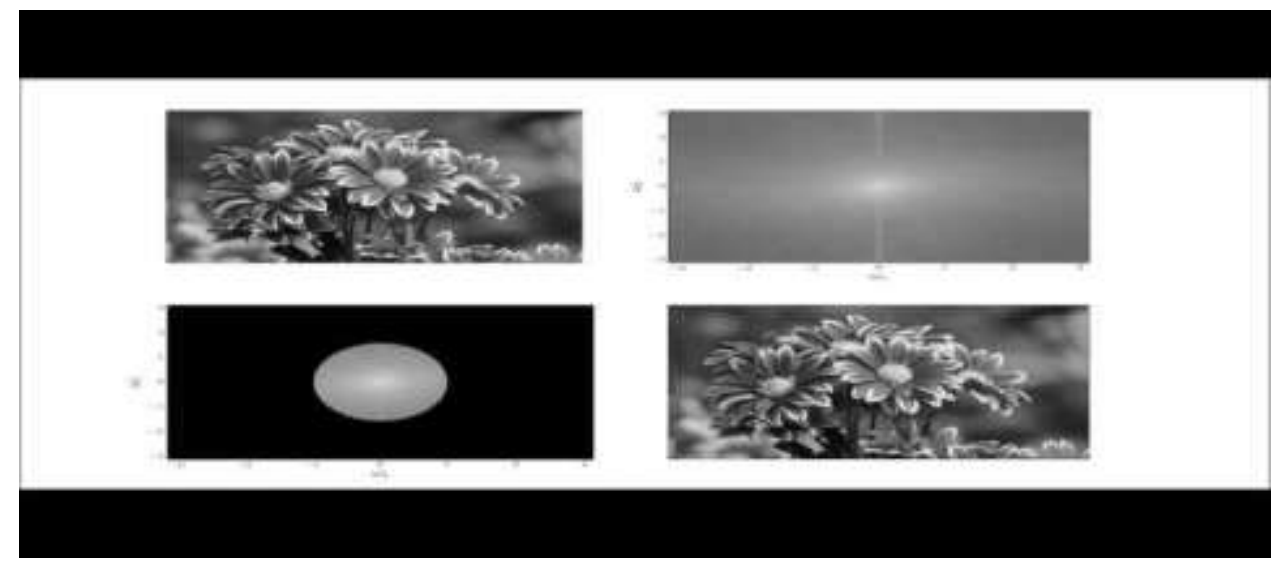

Signal Processing\& LTI system: The Fourier Transform is broadly utilized in the field of Signal Processing. Truth be told, the Fourier Transform is likely the main apparatus for breaking down signals in that whole field. A sign is any waveform (capacity of time). This could be anything in reality - an electromagnetic wave, the voltage across a resistor versus time, the pneumatic force difference because of your discourse (for example a sound wave), or the worth of Apple Stock versus time. Signal Processing then, at that point, is the demonstration of handling a sign to get more helpful data, or to make the sign more valuable. Assume we have a case that acknowledges an info sign and creates a result signal from that. Such a crate can be thought of as a framework:

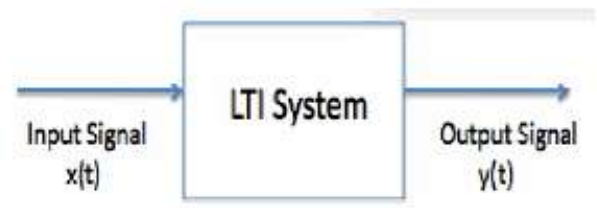

In this system Input is given and Output is generated. Whenever we view the Fourier Transform of the result, we currently realize how the framework responds to each possible frequency. The justification for these returns to the linearity of the Fourier Transform: the drive in time can be considered as a boundless amount of sinusoids at each conceivable recurrence. The result then, at that point, is the amount of the reactions to every recurrence. Fourier Transform envisions the effect of a LTI framework straightforward and the investigation a lot simpler. The Fourier Transform is widely utilized in LTI framework hypothesis, separating and signal handling. Indeed, most of the investigation happens in the recurrence space, making the comprehension of Fourier Theory imperative.

\section{Conclusion}

In this paper we can say that The Fourier Transform settle functions or signs into its method of vibration. It is utilized in planning electrical circuits, tackling differential conditions, signal handling, signal investigation, picture handling and separating. The proposed Fourier transform has a wide scope of help in different areas like power dispersion framework, remote, signal handling, phone producing, mechanical and modern application. In power framework, proposed technique effectively examines the shortcomings, sounds and aggravation. In remote framework, they recognize the commotion and effectively working out the misfortunes in same manner. The Fourier transform gives the world a simple and most agreeable strategy for arrangement of inquiries. Fourier transform is additionally recurrence space which address time series examination demonstrated its application in Quantum mechanics, Signal handling, Image Processing also channels, Transformation, and encoding, Data Processing also Analysis and different fields. In this paper showed that Fourier transform can effectively applied in the field of clinical designing thus some more examination yet to beinvestigated.The thought behind Fourier transform is that an element of direct space can be communicated comparably as a complex function of complementary space that is frequency once in a while called Fourier space. 
[1]. Fourier Transform And Its Application In Modulation Techniques Mrs.SupriyaNilesh Thakur Mrs.MeghaKishorKothawadeAssistant Professor, Basic Engineering Science Department,GuruGobind Singh College Of Engineering And Research Centre,Nashik

[2]. LokhandeAmol D., R. L. Shrivastava, and Rashmi R. Shrivastava. \&quot;A Review on Critical Success Factors of Re-Manufacturing.\&quot; International Journal of Entrepreneurship \&amp; Business Environment Perspectives 3, no. 2 (2014): 982.

[3]. Dr. AmolLokhande, Dr. C. Venkateswaran, Dr. M. Ramachandran, C. Vidhya, R. Kurinjimalar, A Study on Various Implications on Reusing in Manufacturing, REST Journal on Emerging trends in Modelling and Manufacturing 7(2) 2021, 63-69.

[4]. Dr. AmolLokhande, Dr. C. Venkateswaran, Dr. M. Ramachandran, S. Chinnasami, T. Vennila, A Review on Various Implications on Re engineering in Manufacturing, REST Journal on Emerging trends in Modelling and Manufacturing Vol: 7(3), 2021,70-75.

[5]. Dr. AmolLokhande, Dr. C. Venkateswaran, Dr. M. Ramachandran, S. Chinnasami, T. Vennila,Recycling Process Impact in Current Scenario Manufacturing: A Study, REST Recent trends in Management and Commerce, Vol: 2(1), 2021: 20-25

[6]. Lokhande, A. D., Shrivastava, R. L., \&Shrivastava, R. R. (2014). A REVIEW ON CRITICAL SUCCESS FACTORS OF RE-MANUFACTURING. International Journal of Entrepreneurship \& Business Environment Perspectives, 3(2), 982

[7]. Shrivastava, R. L, Lokhande, A. D (2016). LINKAGE BETWEEN EFFORT FACTORS AND ORGANIZATIONAL PERFORMANCE: REMANUFACTURING INDUSTRY. International Journal of Management and Research, 3,301-310

[8]. Shrivastava, R. L, Lokhande, A. D (2015). DESIGN OF FRAMEWORK FOR IMPLEMENTATION OF CSFS IN REMANUFACTURING INDUSTRIES FOR PERFORMANCE IMPROVEMENT. Industrial Engineering Journal. 9(7).39-45.

[9]. Shrivastava, R. R,.Lokhande, A. D., \&Shrivastava, R. L.(2015). "IDENTIFICATION AND EVALUATION OF CRITICAL FACTORS FOR PERFORMANCE IMPROVEMENT FOR REMANUFACTURING INDUSTRY" - A PILOT STUDY. International Journal of Applied Engineering Research. 10(11).10328-10335

[10]. Kamble, A. G., R. Venkata Rao, A. S. Potdar, and A. D. Lokhande. \&quot;Prediction and optimization of spur gear pair by response surface method.\&quot; Joumal on Advances in Science and Engineering, Section B 1 (2010): 23-28.

[11]. Engineering Applications Of The Motion-Group Fourier Transform Gregory S. ChirikjianAnd Yunfeng Wang.

[12]. Fourier Transforms And Its Applications In Engineering Field U S Hegde, Uma S , Aravind P N , Malashri S.

[13]. Applications Of Fourier Transform In Engineering Field Prof. PoonamRajvardhanPatil , Prof. Shankar Akaram Patil.

[14]. The Fourier Transform And Its Applications Prof. Brad Osgood Electrical Engineering Department Stanford University.

[15]. A Brief Study On Fourier Transform And Its Applications NishaChittora, Deepika Babel.

[16]. A Review Of Research Papers On Fourier Transforms \& Statistical Fourier Analysis Mrs. MamtaKansal, Assistant Professor, Department Of Applied Mathematics, Gzs Campus College Of Engg. \&Tech.,Bathinda.(Mrsptu, Bathinda, Punjab, India).

[17]. Applications Of The Fourier Series Matt Hollingsworth.

[18]. Fourier Series And Their Applications RuiNiu.

[19]. Real Time Application Of Fourier Transforms S.ShenbagaEzhil Department Of Mathematics, Amet University, Chennai, India.

[20]. The Fast Fourier Transform And Its Applications Gillian Smith.

[21]. Fourier Series,Fourier Transform And Their Applications To Mathematical Physics Valery Serov.

[22]. Applications Of The Fractional Fourier Transform Cary Yang Carnegie Mellon University.

[23]. Barber, Kevin D., Frank W. Dewhurst, R. L. D. H. Burns, and J. B. B. Rogers. "Business- process modelling andsimulation for manufacturing management: A practical way forward." Business Process ManagementJournal (2003).

[24]. Chan, K. K., and Trevor A. Spedding. "An integrated multidimensional process improvement methodology formanufacturing systems." Computers \& Industrial Engineering 44, no. 4 (2003): 673-693. 